\title{
Physical activity on prescription at the time of stroke or TIA diagnosis - a patient perspective
}

Ann-Sofie, Birgitta Jansson', Gunnel Carlsson²

1 Occupational therapy and Physiotherapy unit, Sahlgrenska University Hospital, Gothenburg, Sweden 2 Department of Clinical Neuroscience, Institute of Neuroscience and Physiology, Sahlgrenska Academy at University of Gothenburg, Sweden

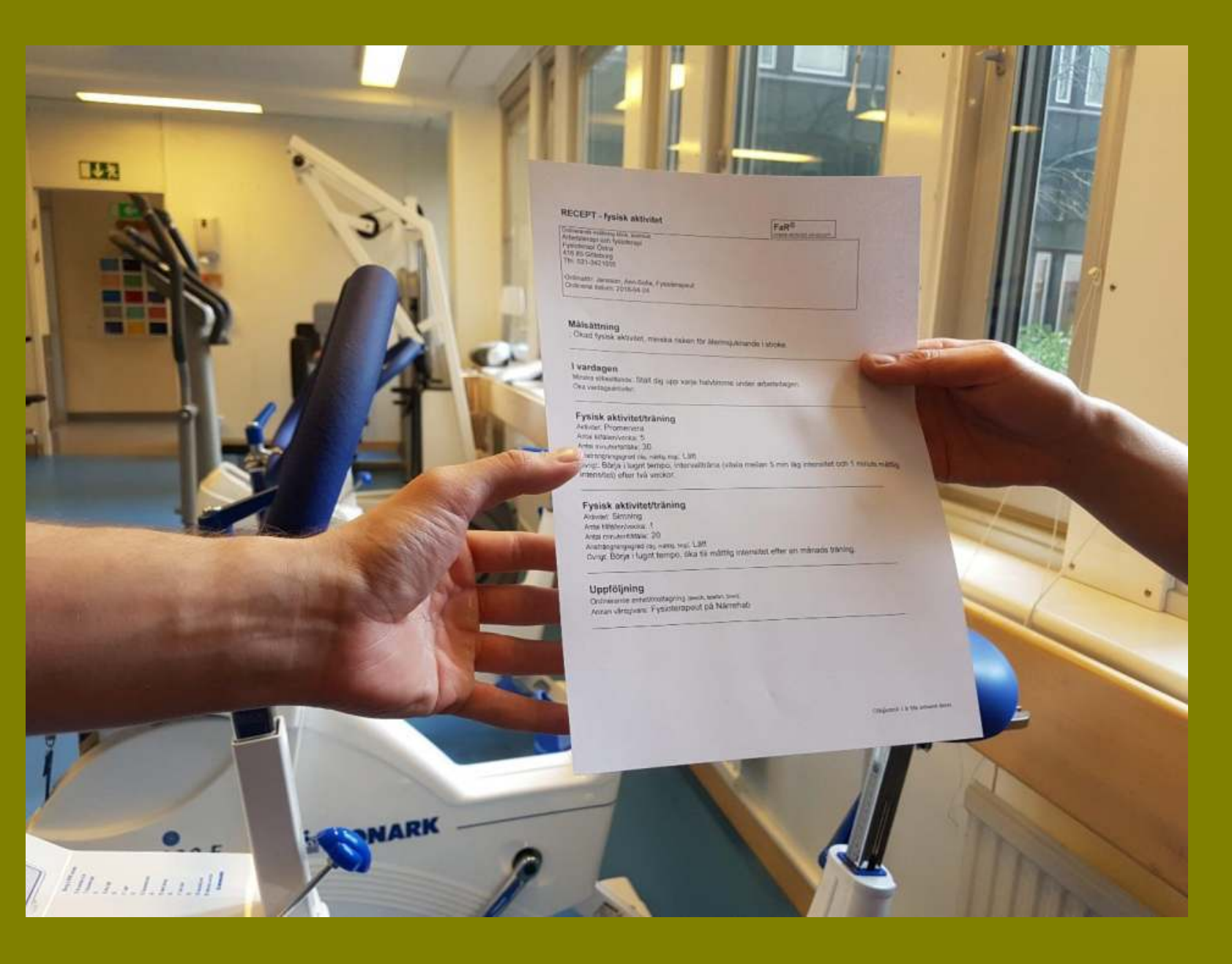

Conclusion: The study highlights the importance of patient participation and individualization when carrying out activities intended to affect lifestyle regarding physical activity. Important clinical implications are the need of reflection on how prescription on physical activity is implemented in acute stroke care, as well as the need to ensure proper follow-up; this to create good conditions for long-term effects.

Background: It is well known that physical activity can improve health. Research has also shown that physical activity on a moderate or high level can prevent recurrent stroke. Despite this quite a lot of individuals who have suffered from stroke have an insufficient level of physical activity.

\section{Change of lifestyle:}

There are many different ways to help individuals to change lifestyle regarding physical activity and Physical activity on Prescription is one of them. Physical activity on Prescription is a recipe with individualized instructions about sort and amount of physical activity.

Purpose: The purpose of this study was to examine how individuals experience getting Physical activity on Prescription at the time of stroke or TIA diagnosis and to explore various factors affecting the ability to follow the recipe after discharge from hospital.
Context of the study: The study was conducted at three stroke units at Sahlgrenska University Hospital, Gothenburg, Sweden. The hospital stay for patients with stroke varies greatly, from a few days to weeks, and for TIA only a couple of days.

Method: Ten indepth interviews were held with patients who had been admitted to a stroke unit and been diagnosed with stroke or TIA. The result was analyzed with qualitative content analysis and is reported with one overall theme and different underlying categories.
Figure 1. An overview of the theme and the main categories and categories that emerged as the result of the analysis of the interviews $[n=10]$.

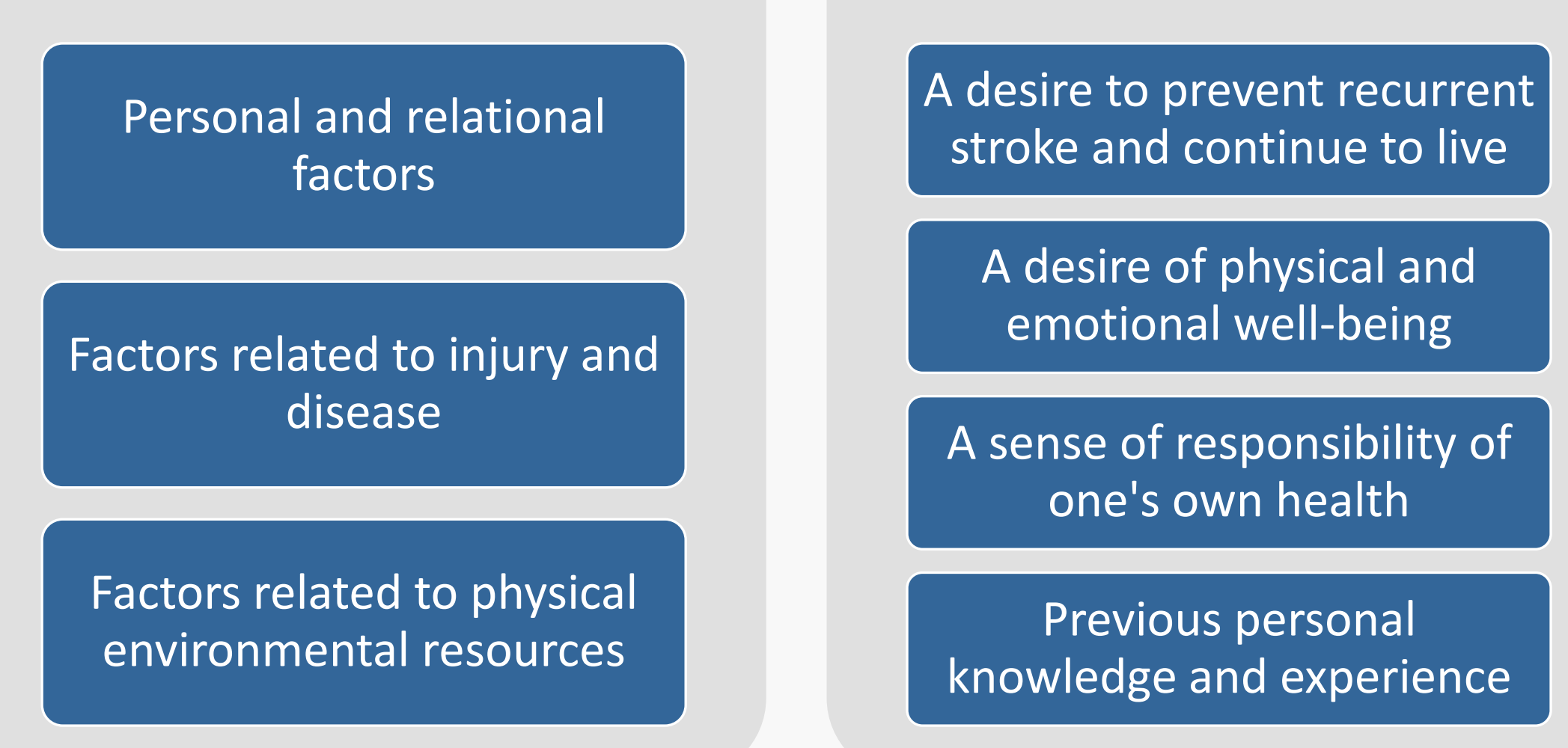

Implications for rehabilitation: Healthcare providers in acute stroke care need to:

1) Reflect on when and how Physical activity on Prescription is prescribed, 2) create opportunities for patient participation, 3) adapt the prescription on the basis of each individual and 4) establish effective procedures to ensure proper follow-up after discharge from hospital. 